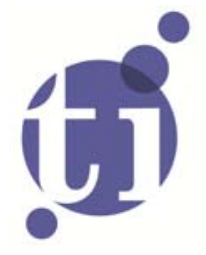

\title{
Biodiversity Prospecting over Time and under Uncertainty: A Theory of Sorts
}

\author{
Amitrajeet A. Batabyall \\ Peter Nijkamp2
}

1 Rochester Institute of Technology, United States of America;

2 Faculty of Economics and Business Administration, VU University Amsterdam, and Tinbergen Institute, The Netherlands. 
Tinbergen Institute is the graduate school and research institute in economics of Erasmus University Rotterdam, the University of Amsterdam and VU University Amsterdam.

More TI discussion papers can be downloaded at http://www.tinbergen.nl

Tinbergen Institute has two locations:

Tinbergen Institute Amsterdam

Gustav Mahlerplein 117

1082 MS Amsterdam

The Netherlands

Tel.: +31(0)205251600

Tinbergen Institute Rotterdam

Burg. Oudlaan 50

3062 PA Rotterdam

The Netherlands

Tel.: +31(0)10 4088900

Fax: $+31(0) 104089031$

Duisenberg school of finance is a collaboration of the Dutch financial sector and universities, with the ambition to support innovative research and offer top quality academic education in core areas of finance.

DSF research papers can be downloaded at: http://www.dsf.nl/

Duisenberg school of finance

Gustav Mahlerplein 117

1082 MS Amsterdam

The Netherlands

Tel.: +31(0)20 5258579 


\title{
Biodiversity Prospecting Over Time and Under Uncertainty:
}

\author{
A Theory of Sorts ${ }^{1}$
}

by

\section{Amitrajeet A. Batabyal ${ }^{2}$}

and

Peter Nijkamp ${ }^{3}$

Batabyal acknowledges financial support from the Gosnell endowment at RIT. The usual disclaimer applies.

2

Department ofEconomics, Rochester Institute ofTechnology, 92 Lomb Memorial Drive, Rochester, NY 14623-5604, USA. Internet aabgshi(:V,rit.edu

3

Department of Spatial Economics, Free University, De Boelelaan 1105, 1081 HV Amsterdam, and Tinbergen Institute, The Netherlands. Internet pnijkamp(a)vu.nl 


\title{
Biodiversity Prospecting Over Time and Under Uncertainty:
}

\section{A Theory of Sorts}

\begin{abstract}
Biodiversity prospecting refers to the exploration of the commercial value of genetic and biochemical resources. In this chapter, we study a drug producing pharmaceutical firm (PF) that searches for potentially useful chemicals made by wild organisms in a conservation area. This PF is able to assign quality levels to the wild organisms in the conservation area. Organism quality is a proxy for the potential usefulness of the chemicals in an organism. At each date, our PF must decide whether to search for a new wild organism with a certain quality or to produce the drug in question with an extant wild organism with its own quality. Our theoretical analysis leads to four results. First, we show that if our PF discards a wild organism with a certain quality at a point in time then it will never use this same organism at a subsequent point in time. Second, we show that if our PF uses a particular organism with its quality at a point in time then it will continue to produce the drug with the chemicals from this organism at all later points in time. Third, we show that there is a threshold level of organism quality and that our PF's optimal policy involves using (discarding) all organisms with quality above (below) this threshold. Finally, we study the impacts of increases in an exogenous income source and the discount factor on our PF's threshold quality.
\end{abstract}

Keywords: Biodiversity Prospecting, Pharmaceutical Firm, Search, Time, Uncertainty

JEL Codes: D81, D83, I12 


\section{Introduction}

\subsection{Preliminaries}

The notion of biological diversity or biodiversity has now become a fashionable concept. This concept refers to the variability among living organisms from all terrestrial and marine sources and from the ecological complexes of which these organisms are a part (Nunes et al., 2003). Biodiversity itself can be of various types such as genetic, species, ecosystem, and functional. The loss of biodiversity is generally considered to be very costly from a societal standpoint and hence many studies have now attempted to assess the economic value ofbiodiversity (Nunes and Nijkamp, 2011). In this regard, a comparative, meta-analytic review of the economic valuation ofbiodiversity can be found in Nijkamp et al. (2008).

Over the past couple of years, several studies have been devoted to the economic analysis of genetic diversity in the context of the commercial search among genetic codes contained in living organisms in order to develop chemical compounds of industrial and pharmaceutical value in agricultural, industrial, and medical applications (see Simpson eta!.,1996, Swanson 1996, and Grifo eta!.,1997). This state of affairs has given rise to intriguing questions about the willingness to pay by biotechnological companies for genetic diversity as inputs into commercial products such as anticancer drugs (see Macilwain 1998, Sonner 1998, Neto and Dickson 1999, and ten Kate and Laird 1999).

An interesting recent survey article on the value of conserving genetic resources for research and development (R\&D) is contained in Sarr et al. (2008). These authors assess the extent to which society is able to invest now in order to prepare for future risks and uncertainties in the arrival of various biological and medical contingencies. Such issues have given rise to a new strand of 
literature on what is now known as biodiversity prospecting or bioprospecting.

Specifically, biodiversity prospecting refers to "the exploration of biodiversity for commercially valuable genetic and biochemical resources" (Reid eta!., 1993a, p.l). As noted by Eisner (1989, 1992), Reid eta!. (1993b), and others, ecologists, environmentalists, and taxonomists have been saying for some time that it should be possible to justify the conservation of biodiversity on the basis of its many pharmaceutical and other commercial applications. Even so, interest among pharmaceutical firms in particular in biodiversity prospecting has been muted until the completion of the now prominent agreement between Costa Rica's National Biodiversity Institute (InBio) and the United States based pharmaceutical giant Merck and Company.

The September 1991 agreement between InBio and Merck contained two key provisions. First, InBio would provide Merck with a whole host of chemical extracts from wild plants, insects, and micro-organisms from Costa Rica's conserved wildlands for Merck's drug screening program. In turn, Merck would provide InBio with a research and sampling budget ofUS\$1,135,000 and royalties on commercial products arising from the InBio provided chemical extracts. Laird (1993) and Sittenfeld and Gamez (1993) rightly note that this agreement has proved to be a watershed event in the history of biodiversity prospecting. In addition, this agreement has given rise to significant interest in designing "win-win"biodiversity prospecting contracts that, inter alia,provide an explicit economic rationale for the conservation of biodiversity in many different parts of the world.

As our thinking on the subject ofbiodiversity prospecting has progressed, our understanding of the merits and demerits of this activity has become nuanced. Therefore, it is fair to say that in the context of the conservation of biodiversity, broadly speaking, the contemporary literature in economics consists of a group of researchers who are positively inclined towards biodiversity 
prospecting, a second group that is largely neutral about the utility of biodiversity prospecting, and a third group that sees little of value in biodiversity prospecting. We now summarize the findings of representative contributions from each of these three groups.

\subsection{Review of the literature}

\subsubsection{The positive perspective}

The Uruguay round of the General Agreement on Tariffs and Trade (GATT) proposed that, inter alia, trade related intellectual property rights or TRIPS be conferred on international firms on a whole host oflife forms and on biotechnology. Given this background, Bhat (1996) points out that establishing intellectual property rights to products derived from genetic and biochemical resources is necessary but not sufficient for biodiversity prospecting and the survival of three kinds of biological resources. He urges developing nations to create institutions and policies that will enable local communities to receive the "benefits of biodiversity conservation and prospecting" (Bhat, 1996, p. 205).

What lessons can one learn from the successes and the eventual failure of Shaman Pharmaceuticals, a once promising player in the biodiversity prospecting market? Clapp and Crook (2002) contend that even though Shaman Pharmaceuticals eventually failed, the key lesson to be learned from this failure is that because of rapid technological change, new models and institutions are needed for drug development from natural products. In addition, it is important to comprehend that "bioprospecting" and "ethnobotanical searches" will continue to be salient activities because "natural products will remain important to drug development" (Clapp and Crook, 2002, p. 79).

Some of the world's most biologically diverse resources are to be found in the tropics. This

much is well known and agreed upon. Kala (2006) focuses on the Himalayan region in northern 
India and notes that it has often not been possible to meet the increasing demand for medicinal plants from both pharmaceutical firms and herbal healers because of several constraints. One such constraint stems from the "specific ecological requirements of many Himalayan medicinal plant species" (Kala, 2006, p. 370). This notwithstanding, this researcher sees value in biodiversity

prospecting and, as such, he discusses ways in which the medicinal plants sector might be developed and managed.

\subsubsection{The neutral perspective}

Mulholland and Wilman (2003) conduct an interesting intertemporal analysis of the properties of a biodiversity prospecting contract between a host nation and a pharmaceutical finn. As expected, in this analysis, the host nation's stock of biodiversity and genetic information are the key inputs in the production of high quality samples. These authors demonstrate that even with complete property rights, contracts are second best because it is impossible to perfectly monitor the host nation's inputs in the process of drug discovery. More generally, it is shown that contracts "vary due to the different degrees of observability of host-country inputs, and incomplete or ineffective property rights" (Mulholland and Wilman, 2003, p. 417).

Clearly, biodiversity prospecting can create incentives for the private conservation of what are often known as "biodiversity hotspots." Given this situation, will a market for biological resources give rise to sufficient incentives for private conservation? This pertinent question is analyzed by Di Corato (2007). This researcher develops and studies a market framework and shows that different market equilibria are possible and that these different equilibria have different implications for the extent of conservation. In particular, the "industry structure on the supply side" (Di Corato, 2007, p. 44) is shown to have a fundamental bearing on the private incentive to conserve 
biodiversity.

Ozturk and Ozturk (2008) begin their analysis of the biological screening of medicinal plants in developing countries with two observations. First, they note that the importance of medicinal plants has been increasing over time for both pharmaceutical firms and traditional users. Second, they contend that environmental changes stemming from global warming are likely to have a nontrivial impact on the natural resources of developing countries. In light of these two observations, these authors rightly note that there are threats stemming from the biological screening of medicinal plants. This notwithstanding, Ozturk and Ozturk (2008) clearly state that if the stages and the techniques of pharmacological screening and the differences between natural and synthetic products are well understood then there also exist many opportunities from biodiversity prospecting for pharmaceutical firms in developing nations.

\subsubsection{The negative perspective}

In a prominent paper, Simpson et al. (1996) analyze the valuation of biodiversity for use in pharmaceutical research. The central contention of the authors of this paper is that even though biodiversity prospecting has been much lauded for being an effective mechanism for discovering novel pharmaceutical products and for conserving biodiversity, theoretical analysis warrants a much more cautious approach. Specifically, these authors value the marginal species on the basis of its incremental contribution to the likelihood of making a commercial discovery. It is shown that even under favorable assumptions, the upper bound on the value of the marginal species is modest. This fmding is then extended to valuing the marginal hectare ofhabitat. This exercise leads these authors to conclude that "the incentives for habitat conservation generated by private pharmaceutical research are...at best, very modest" (Simpson et a/.,1996, p. 163). 
The success of arguments promoting biodiversity prospecting as a way of conserving biodiversity ultimately depends on the value ofbiodiversity for use in new pharmaceutical research. Given this state of affairs, Craft and Simpson (2001) attempt to estimate the above mentioned value using two models of competition among differentiated products. Analysis of both models confirms a key fmding in the Simpson et al. (1996) paper discussed in the previous paragraph. Specifically, it is shown that the "value to private researchers of the 'marginal species' is likely to be small" (Craft and Simpson, 2001, p. 1). This negative fmding notwithstanding, these researchers stress that the models being analyzed have very different implications for the social value of biodiversity. Therefore, these researchers conclude their analysis with a plea for a better understanding ofthe true meaning ofbiodiversity.

Extending the arguments made in the two papers discussed in the preceding two paragraphs, Sedjo and Simpson (2005) focus on the nexuses between investments in biodiversity prospecting and incentives for biodiversity conservation. Their analysis leads these researchers to contend that investments in biodiversity prospecting are unlikely to increase incentives for conservation by much. This contention is explained by noting that if the value of the marginal species were noteworthy then investments already ought to have been made to exploit this species. On the other hand, ifthis value is not noteworthy then it is unlikely that additional investments in biodiversity prospecting will lead to any substantial increase in the incentives for biodiversity conservation. This line of reasoning leads these authors to conclude that if we believe that biodiversity is salient then strategies more effective than biodiversity prospecting need to be found to ensure its conservation.

\subsection{Implications and the contributions of this chapter}

Our review of the literature in sections 1.2.1-1.2.3 above leads to three conclusions. First, 
the various studies we have discussed have certainly advanced our understanding of the many nexuses between biodiversity prospecting on the one hand and biodiversity conservation on the other. Second, we see that the picture concerning the desirability of biodiversity prospecting as a tool for promoting conservation is mixed. Finally, even though biodiversity prospecting can, at least in some circumstances, be a desirable tool for promoting biodiversity conservation, there are virtually no theoretical studies of biodiversity prospecting that explicitly account for the facts that this process takes place over time and under uncertainty.

Therefore, to fix ideas and to provide answers to previously unstudied questions about biodiversity prospecting, in this chapter, we model and analyze the activities of a drug producing pharmaceutical firm (PF) that searches for potentially useful chemicals made by wild organisms in a specific conservation area. This PF is able to assign quality levels to the wild organisms in the conservation area. From a drug production standpoint, organism quality is a proxy for the possible usefulness of the underlying chemicals in an organism. At each date, our PF must decide whether to search for a new wild organism with a certain quality or to produce the drug in question with an extant wild organism with its own quality.

Our theoretical analysis sheds light on the following four hitherto unstudied questions concerning biodiversity prospecting. First, if our PF discards a wild organism with a certain quality at a specific point in time then is it ever optimal for it to use this same organism at a future date? Second, if our PF uses a particular organism with its quality at a specific point in time then ought it to continue to produce the drug in question with the chemicals from this organism at all subsequent points in time? Third, is there a threshold level of organism quality such that an optimal course of action requires our PF to use (discard) all organisms with qualities that are above (below) 
this threshold? Finally, what are the impacts of increases in an exogenous income source and the discount factor on our PF's threshold quality?

The rest of this chapter is organized as follows. Section 2 adapts the analysis in McCall (1970) and in Batabyal and Beladi (2010) and delineates a dynamic and stochastic model of biodiversity prospecting by a PF that involves a choice between the search for new wild organisms with distinct qualities and drug production using an extant wild organism with its own quality. ${ }^{4}$ Section 3 provides an analysis of the first question mentioned in the preceding paragraph. Section 4 studies the second question stated in the previous paragraph. Section 5 sheds light on the third question from the previous paragraph. Section 6 discusses the preceding paragraph's fourth and last question. Section 7 concludes and then discusses potential extensions of the research described in this chapter.

\section{A Model of Biodiversity Prospecting}

Consider the decision problem faced by a PF that operates in a dynamic and stochastic environment. In our model, time is discrete, the discount factor at time $t$ is pt, our PF's profit at time $t$ is $\mathrm{n}(\mathrm{t})$, and its risk-neutral objective function is $;=\mathrm{oPt} 1 \mathrm{t}(\mathrm{t})$. To keep the following analysis straightforward, we assume that our PF is unable to either borrow or to lend and hence its profit in any time period is equal to the income it generates in this same time period.

At time $t$, if our PF uses the chemicals in a wild organism ${ }^{5}$ of quality $q(t)$ then we suppose that it can generate income given by $i(t)=q(t)$. Also, at time $t=O$, without loss of generality, we suppose that our PF begins drug production with the chemicals from a wild organism of quality

\footnotetext{
See Ljungqvist and Sargent (2005) for a lucid exposition of the McCall (1970) model.

5
}

For concreteness, the reader may want to think of this wild organism as a wild plant species. 
$q(O)=O$. From then on, at each date, this PF can either produce the drug in question using the chemicals from any one of the wild organisms it has already found or it can spend this time period searching for a new wild organism. In each time period in which our PF searches for the chemicals from a new wild organism, it obtains an independent realization from a time invariant cumulative distribution function $G(q)$ which is defmed over the bounded and closed interval [O,q].

The decision problem confronting our PF at each date is to determine whether to search for a new wild organism or to produce the drug in question with the chemicals from one of the wild organisms it has already found. Because there is no borrowing or lending in our model, at every date, profit equals current income which, in turn, equals the value/quality of the drug. In symbols, we have $1 t(t)=i(t)$. The reader will note that at every date, our PF has a significant choice before it that affects the quality of the input (chemicals from a wild organism) that is actually available to it. In particular, by searching for additional time periods, which is costly in terms of foregone drug production, this PF can potentially ameliorate the quality levels of the chemicals from the various wild organisms that are available to it.

Let us assume that our PF can use the chemicals from any one of the wild organisms that it has found in the past to produce the pertinent drug at any point in time. In addition, in principle, it can also stop producing the drug at any date and go back to searching for new wild organisms. Given this state of affairs, our first task now is to formulate our PF's maximization problem recursively. To do so, we shall use the techniques of stochastic dynamic programming. ${ }^{6}$

Let $q m(t)=\max \left\{t^{\prime} E[0, t]\right\} q\left(t^{1}\right)$ denote the wild organism with the highest quality available to our PF at time $t$. Note that this PF will not use the chemicals from any wild organism with quality

See Ross (1983), Puterman (2005), and Acemoglu (2009) for textbook treatments of stochastic dynamic programming. 
in the set $\{q(O), \ldots, q(t)\}$ that is not equal to $q m(t)$ and hence we can think of $q m(t)$ as the payoff relevant state variable at time $t$. Suppose our PF searches at time $t$. Then it produces the drug in question with the chemicals from the wild organism of quality $q m(t)$ and receives the continuation value that we shall represent with $V\{q m(t+1)=q m(t)\}$ given that it does not find a new wild organism. Once again, suppose that our PF searches at time $t$. Now, in an alternate scenario, our PF does not produce the drug at time $t$ but it receives the continuation value $V\{q m(t+1)=\max (q m(t), q)\}$ where $q$ is the quality of the chemicals in the wild organism found at timet.

Combining the two observations from the preceding paragraph, our PF's maximization problem can be formulated in a recursive manner. This formulation gives us

$$
V\{q m\}=\max \{q m+p V(q m), p E[V\{\max (q m, q)\}]\},
$$

where $E[\cdot]$ is the expectation operator. Using integrals, the expectation on the RHS of(1) can be expressed in a more suitable manner. We get

$$
V\{q \mathrm{~m}\}=\max \left\{\mathrm{q} m+p V(q m), \mathrm{p} \boldsymbol{g}_{0}\{\max (q m, \mathrm{q})\} \mathrm{dG}(\mathrm{q})\right\}
$$

This completes the task of formulating our PF's maximization problem. We now proceed to study the main properties of this PF's optimal course of action by concentrating on the first question posed in the penultimate paragraph of section 1.3. In particular, if our PF discards a wild organism with a certain quality at a specific point in time then we want to know whether it is ever optimal for it to 
use this same organism to produce the drug in question at a later point in time.

\section{To Use or Not to Use a Previously Discarded Organism}

In order to answer the above query, we must first express this query in mathematical terms. To this end, suppose that our PF has decided that it will not produce the drug in question using the wild organism with quality $q^{1}$ at time $t$. Then, what we want to demonstrate is that it will never use this organism with quality $q^{1}$ at time $t+s$ where $s>O$. Put differently, if our PF discards a wild organism with a particular quality at a certain date, then it will never use this same organism for any possible realization of events between dates $t$ and $t+s$.

To demonstrate this "no use" result, let us assume that our PF decides to use a wild organism with a certain quality when it is indifferent between using this organism and searching for additional periods of time. Also, we assume that the set of wild organisms with distinct qualities found through search are strictly ordered at the top. ${ }^{7}$ We are now ready to provide a proofby contradiction. To this end, suppose that when our PF has access to the set of wild organisms with qualities $q t=\left\{q(O), \ldots, q^{1}, \ldots, q(t)\right\}$, it chooses to search for new wild organisms at time $t$ and that it uses the wild organism with quality $q^{1}$ at time $t+s$ for some $s>O$. Now, since our PF has decided to search at time $t$, we know that the following strict inequality

$$
q \mathrm{~m}(\mathrm{t})+\mathrm{p} V\{q \mathrm{~m}(\mathrm{t})\}<\underset{0}{i j} V\{\max (q \mathrm{~m}(\mathrm{t}), i j)\} d G(i j)
$$

must hold. Also, because our PF uses the wild organism with quality $q^{1}$ at time $t+s$, we have

With this assumption, we are ruling out instances in which there are two maxima so that our PF moves back and forth between using these two wild organisms and a wild organism that is not used at time $t$ but used at time $t+s$. 
$q m(t+s)=q^{1}$. These last two results tell us that the weak inequality

$$
\left.q m(t+s)+p V\{q m(t+s)\}:<: p \int^{l j} \max (q m(t+s), i j)\right\} d G(i j)
$$

also holds.

We know that the wild organism with the highest quality at time $t+s$ is at least as good as the wild organism with the highest quality at time $t$ for any realization of events between times $t$ and $t+s$. In symbols, this means that $q m(t+s):<: q m(t)$. Also, we know that the function $V\{\cdot\}$ is weakly increasing. Given this finding, the inequalities in (3) and (4) together tell us that

$$
q m(t+s)+p V\{q m(t+s)\}>q m(t)+p V\{q m(t)\} .
$$

Since the function $\mathrm{V}\{\cdot\}$ is weakly increasing, we can use the inequality in (5) to determine that $q m(t+s)>q m(t)$. On the other hand, we know that $q^{1}=q m(t+s)$ and $q t=\left\{q(O), \ldots, q,{ }^{1} \ldots, q(t)\right\}$ which implies that $q m(t+s)=q m(t)$ which is plainly not possible. This gives us the contradiction we seek and, as such, we have also demonstrated that in an optimal course of action, our PF never uses a wild organism it has discarded in the past. We now proceed to study a second basic property of our PF's optimal course of action. Recall from the discussion in the penultimate paragraph of section 1.3 that this involves answering the following query. If our PF produces the drug in question with a particular quality wild organism at a specific point in time then ought it to continue to produce the drug with this organism at all succeeding points in time? 


\section{Drug Production Continuity With an Organism in Use}

Suppose our PF uses the wild organism with quality $q^{1}$ at time $t$. Then, some thought ought to convince the reader that the query at the end of the preceding paragraph involves ascertaining whether, for all dates s'z.t, it makes sense for our PF to stop drug production with the wild organism with quality $q^{1}$ and to go back to searching for new wild organisms.

Given that our PF has agreed to produce the drug in question with the wild organism with quality $q^{1}$ at time $t$, it is obvious that $q m(t)=q^{1}$.Also, given the decision to use this organism, the weak inequality

$$
q^{1}+p V\left\{q^{1}\right\}-z \_p \underset{0}{J j}\left\{\max \left(q^{1}, i j\right)\right\} d G(i j)
$$

must hold. SinceourPF doesnotsearchforwildorganismsattime $t+\mathbf{1}$, we get $q m(t+1)=q \quad m(t)=q^{1}$. Hence, from the inequality in (6) it follows that our PF also uses the wild organism with quality $q^{1}$ at time $t+1$. Now, it follows by mathematical induction that our PF will continue to produce the pertinent drug with the wild organism with quality $q^{1}$ for all dates $s^{\prime} z . t$ and will never go back to searching for new wild organisms. The next topic on the agenda is to study a third basic property of our PF's optimal course of action. Recall that this involves ascertaining whether there is a threshold level of organism quality such that an optimal course of action requires our PF to use (discard) all organisms that are above (below) this threshold.

\section{The Threshold Level of Organism Quality}

Before moving on, let us briefly summarize the implications of our analysis thus far in sections 2 through 4. First, in section 3 we demonstrated that our PF never uses a discarded wild 
organism from the past and hence there is no loss of generality in assuming that the payoff relevant state variable is the most recent wild organism that is found. Second, in section 4 we established that our PF never goes back to searching for wild organisms once it has decided to produce the drug in question with the chemicals from a particular wild organism. This means that the value to our PF from deciding to use a wild organism with quality q-for drug production--can be expressed as $\operatorname{vuse}(q)=q /(1-\mathrm{p})$. Third, given these two points, our PF's maximization problem can be formulated as a stationary or time independent problem in which the function $\mathrm{V}\{\cdot\}$ has a piecewise linear form. ${ }^{8}$

With this summary in place, let us analyze the case in which our PF is not producing the drug in question and it receives an exogenous amount of income denoted by ie. ${ }^{9}$ Now, using an approach similar to that employed in section 2, we want to state our PF's maximization problem and to examine the potential existence of a threshold level of wild organism quality. To begin our analysis, we suppose that $i e<q$. If this were not the case then it would never be optimal for our PF to use any wild organism with its distinct quality and the trivial optimal solution to our PF's maximization problem would involve always searching for wild organisms at all points in time.

Given the summary in the first paragraph of this section, when $i e<q$, without any loss of generality, the maximization problem for our PF can be recursively written as

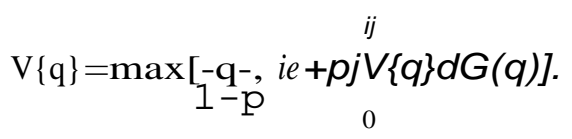

Since $V\{\cdot\}$ is the maximum of a constant function and a linear function, $V\{\cdot\}$ is itself a piecewise

See equation 16.28 in Acemoglu (2009, p. 558) for additional details on this point.

9

We shall not concern ourselves with the source of this income but it could arise, for instance, from licensing activities. 
linear function. This tells us that our PF's optimal policy does involve a threshold rule. In other words, there exists a threshold level of organism quality $T$ such that our PF decides to use all organisms with qualities that are above $T$ and it discards all organisms with qualities $q<T$ and continues to search for new wild organisms. Mathematically, the pertinent threshold $T$ satisfies

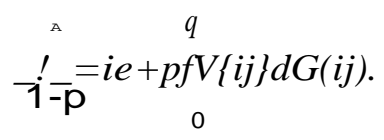

For a wild organism with quality $q<T$, we have $\mathrm{V}\{\mathrm{q}\}=\mathrm{V}\{\mathrm{t}\}$ and for a wild organism with quality $q>T$, we have $V\{q\}=q /(1-p)$. With these two pieces of information, (8) tells us that

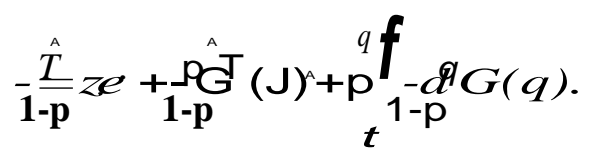

Now, if we subtract the identity

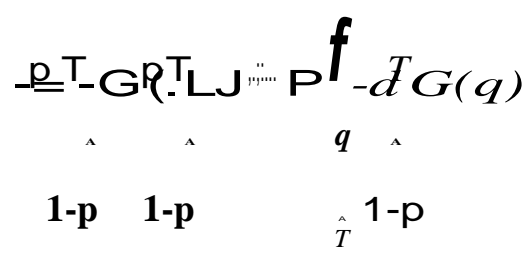

from both sides of (9), then the threshold quality level $\boldsymbol{t}$ solves

$$
T=i e+\underset{\mathbf{1}-\mathbf{p}}{P}-f\left(q-i^{\prime}\right) d G(q)
$$

We can think of the LHS of (11) as the cost of foregoing drug production with the wild organism with quality $T$. In contrast, the RHS of (11) is the expected benefit to our PF from one 
more round of searching for wild organisms. Clearly, for the organism with threshold quality level $f^{\prime}$, these two values have to equal each other. In words, this simply means that for the wild organism with threshold quality level $\mathrm{f}^{\prime}$, our PF is indifferent between the two actions of producing the drug in question and continuing the search for wild organisms.

We have now demonstrated that in the modeling setup of this chapter, there exists a threshold level of organism quality $T$ in the sense that an optimal course of action requires our PF to use (discard) all organisms with qualities that are above (below) this threshold. Also, we have solved for this threshold quality level in (11). We now proceed to our last task in this chapter and this entails an analysis ofthe effects of increases in the exogenous income ie and the discount factor $\mathbf{p}$ on our PF's threshold organism quality level f'.

\section{Changes in the Threshold Level of Organism Quality}

We first concentrate on the exogenous income $i e$. Let us represent the RHS of (11) with the function $C(i e, i)$. Observe that $\left((\cdot ;)\right.$ is decreasing in the threshold organism quality level $f^{\prime}$. Also, we know that $((\mathrm{ie}, \mathrm{O})>\mathrm{O}$ and, by assumption, that $((i e, i j)=i e<4 \cdot$ This tells us that the function ((ie;) crosses the 45 degree line. Since this is a decreasing function, it crosses the 45 degree line exactly once and hence the equation $T=$ ( (ie'i) has a unique solution $T E(0, q)$ for any value of exogenous income $i e^{\prime}$ Inaddition, because the function ( $\left(i e^{\prime} i\right)$ is increasing in $i e^{\prime}$ the unique solution $T$ is also increasing in ie. This tells us that the threshold organism quality level $T$ rises as the exogenous income ie rises. From an intuitive standpoint, this result is telling us the following. When our PF receives additional benefits from searching for wild organisms, it has a greater incentive to continue to search and hence it requires a higher threshold to use an organism and produce the drug in question. 
Given the discussion in the preceding paragraph, the effect of an increase in the discount factor $\mathrm{p}$ is quite simple to determine and therefore we provide only an intuitive delineation of the relevant effect. The reader should note that an increase in $\mathrm{p}$ makes our PF more patient or more concerned about the future. Hence, it should be clear to the reader that this increased patience or concern for the future increases the threshold level of organism quality $T$. In their analysis of the value of the marginal species, Simpson et al. (1996) do not consider the effects of discounting. However, they claim that the introduction of discounting is likely to strengthen their fmding that the upper bound on the value ofthe marginal species is modest. The implication is that this, most likely, will further discourage biodiversity prospecting. Our analysis of this chapter's model ofbiodiversity prospecting with discounting leads to a somewhat more specific result. We fmd that an increase in the discount factor raises the wild organism use threshold and hence this is likely to delay drug production. This concludes our discussion of the fourth and last question of this chapter.

\section{Conclusions}

In this chapter, we examined the activities of a PF that, at each date, had to decide between producing the drug in question with an existing organism with its distinct quality and searching for new wild organisms. Our examination shed light on four hitherto unstudied questions in the existing literature on biodiversity prospecting. First, we demonstrated that if our PF discards a wild organism with a certain quality at a specific point in time then it will never use this same organism at a subsequent point in time to produce the drug in question. Second, we determined that if our PF decides to engage in drug production with an organism of a certain quality at a specific point in time then it will continue to produce the drug in question with the chemicals from this organism at all subsequent points in time. Third, we showed that there exists a threshold organism quality level and 
that our PF's optimal policy involves using (discarding) all organisms with qualities above (below) this threshold. Finally, we analyzed the effects of increases in an exogenous income source and the discount factor on our PF's threshold level of organism quality.

The analysis in this chapter can be extended in a number of directions. Here are two suggestions for extending the research described here. First, Simpson et al. (1996, p. 165) have rightly noted that all theoretical models are built on a number of simplifying assumptions and our model certainly fits this description. Specifically, in the model of this chapter, we have significantly compressed the amount of time it takes a PF to make a determination of the quality of a wild organism. In reality, this quality determination exercise is an elaborate process involving the active participation of several trained groups of individuals. Therefore, it would be useful to analyze the wild organism quality determination aspect of biodiversity prospecting in greater detail.

Second, we have focused exclusively on the private incentives facing a PF. Clearly, the social incentives for biodiversity conservation are almost certainly higher than the private incentives. Therefore, it would be instructive to analyze a model that explicitly accounts for the fact that consumer surplus from new drug development may well exceed the profits of a PF by a large margin. Studies of biodiversity prospecting that incorporate these features ofthe problem into the analysis will provide additional insights into an activity that many believe can play a positive role in conserving some of our most valuable natural resources. 


\section{References}

Acemoglu, D. 2009. Introduction to Modern Economic Growth. Princeton University Press, Princeton, NJ.

Batabyal, A.A., and Beladi, H. 2010. A model of entrepreneurial activity with two actions over time and under uncertainty, Letters in Spatial and Resource Sciences, 3, 45-54.

Bhat, M.G. 1996. Trade-related intellectual property rights to biological resources: Socioeconomic implications for developing countries, Ecological Economics, 19, 205-217.

Clapp, R.A., and Crook, C. 2002. Drowning in the magic well: Shaman pharmaceuticals and the elusive value of traditional knowledge, Journal of Environment and Development, 11, 79102.

Craft, A.B., and Simpson, R.D. 2001. The value of biodiversity in pharmaceutical research with differentiated products, Environmental and Resource Economics, 18, 1-17.

Di Corato, L. 2007. Bioprospecting and market for biodiversity conservation, International Journal of Ecological Economics and Statistics, 7, 44-67.

Eisner, T. 1989. Prospecting for nature's chemical riches, Issues in Science and Technology, 6, 31-

34.

Eisner, T. 1992. Chemical prospecting, in F.H. Bormann and S.R. Kellert, (Eds.), Ecology, Economics, and Ethics, 196-202. Yale University Press, New Haven, CT.

Grifo, F., Newman, D., Fairfield, A.S., Bhattacharya, B., and Grupenhoff, J.T. 1997. The origin of prescription drugs, in F. Grifo, J. Rosenthal, and T. Lovejoy, (Eds.),BiodiversityandHuman Health, 131-163. Island Press, Washington, DC.

Kala, C.P. 2006. Problems and prospects in the conservation and development of the Himalayan 
medicinal plants sector, International Journal of Sustainable Development, 9, 370-389.

Laird, S.A. 1993. Contracts for biodiversity prospecting, in W.V. Reid, S.A. Laird, C.A. Meyer, R. Gamez, A. Sittenfeld, D.H. Janzen, M.A. Gollin, and C. Juma, (Eds.), Biodiversity Prospecting, 99-130. World Resources Institute, Washington, DC.

Ljungqvist, L., and Sargent, T.J. 2005. Recursive Macroeconomic Theory. MIT Press, Cambridge, MA.

Macilwain, C. 1998. Bid to block Yellowstone enzymes deal, Nature, 392, 117.

McCall, J. 1970. Economics ofinformation andjob search, Quarterly Journal of Economics, 84, 113-126.

Mulholland, D.M., and Wilman, E.A. 2003. Bioprospecting and biodiversity contracts, Environment and Development Economics, 8, 417-435.

Neto, R.B., and Dickson, D. 1999. \$3million deal launches major hunt for drug leads in Brazil, Nature, 400, 302.

Nijkamp, P., Vindigni, G., and Nunes, P.A.L.D. 2008. Economic valuation of biodiversity: A comparative study, Ecological Economics, 67,217-231.

Nunes, P.A.L.D., and Nijkamp, P. 2011. Biodiversity: Economic perspectives, Unpublished Manuscript, Free University, Amsterdam.

Nunes, P.A.L.D., Van Den Bergh, J.C.J.M., and Nijkamp, P. 2003. The Ecological Economics of Biodiversity. Edward Elgar, Cheltenham, UK.

Ozturk, N., and Ozturk, Y. 2008. Opportunities and threats for biological screening of medicinal plants: Importance for the pharmaceutical industries in developing countries, World Review of Science, Technology, and Sustainable Development, 5, 124-139. 
Puterman, M.L. 2005. Markov Decision Processes. Wiley, Hoboken, NJ.

Reid, W.V., Laird, S.A., Gamez, R., Sittenfeld, A., Janzen, D.H., Gollin, M.A., and Juma, C. $1993 a$. A new lease on life, in W.V. Reid, S.A. Laird, C.A. Meyer, R. Gamez, A. Sittenfeld, D.H. Janzen, M.A. Gollin, and C. Juma, (Eds.), Biodiversity Prospecting, 1-52. World Resources Institute, Washington, DC.

Reid, W.V., Laird, S.A., Meyer, C.A., Gamez, R., Sittenfeld, A., Janzen, D.H., Gollin, M.A., and Juma, C. Eds. 1993b. Biodiversity Prospecting. World Resources Institute, Washington, DC.

Ross, S.M. 1983. Introduction to Stochastic Dynamic Programming. Academic Press, San Diego, CA.

Sarr, M., Goeschl, T., and Swanson, T. 2008. The value of conserving genetic resources for R\&D: A survey, Ecological Economics, 67, 184-193.

Sedjo, R.A., and Simpson, R.D. 2005. Investments in biodiversity prospecting and incentives for conservation, Discussion Paper, Resources for the Future, Washington, DC.

Simpson, R.D., Sedjo, R.A., and Reid, J.W. 1996. Valuing biodiversity for use in pharmaceutical research, Journal of Political Economy, 104, 163-185.

Sittenfeld, A., and Gamez, R. 1993. Biodiversity prospecting by INBio, in W.V. Reid, S.A. Laird, C.A. Meyer, R. Gamez, A. Sittenfeld, D.H. Janzen, M.A. Gollin, and C. Juma, (Eds.), Biodiversity Prospecting, 69-98. World Resources Institute, Washington, DC.

Sonner, S. 1998. Suit tries to block 'bioprospecting' in Yellowstone, Idaho News, March 6.

Swanson, T. 1996. The reliance of northern economies on southern biodiversity, Ecological Economics, 17, 1-8.

ten Kate, K., and Laird, S.A. Eds. 1999. The Commercial Use of Biodiversity. Earthscan, London, 
UK. 